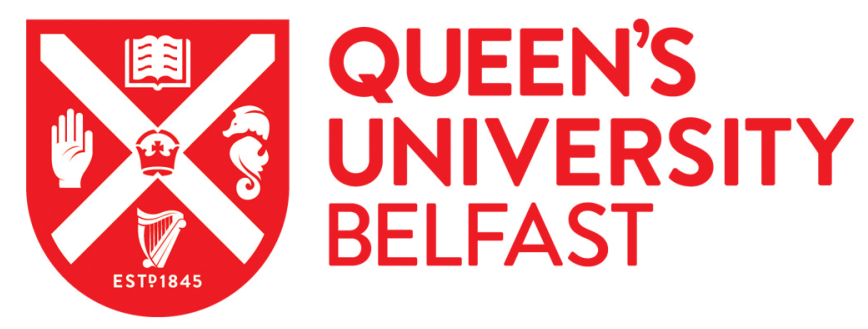

\title{
A Monte Carlo simulation model to assess volunteer response times in a Public Access Defibrillation scheme in Northern Ireland
}

\author{
Marshall, A., Cairns, K., Kee, F., Moore, M. J., Hamilton, A. J., \& Adgey, A. A. J. (2006). A Monte Carlo \\ simulation model to assess volunteer response times in a Public Access Defibrillation scheme in Northern \\ Ireland. 783-788. Paper presented at 19th IEEE Symposium on Computer-Based Medical Systems, Salt Lake \\ City, United States.
}

\section{Queen's University Belfast - Research Portal:}

Link to publication record in Queen's University Belfast Research Portal

\footnotetext{
General rights

Copyright for the publications made accessible via the Queen's University Belfast Research Portal is retained by the author(s) and / or other copyright owners and it is a condition of accessing these publications that users recognise and abide by the legal requirements associated with these rights.

Take down policy

The Research Portal is Queen's institutional repository that provides access to Queen's research output. Every effort has been made to ensure that content in the Research Portal does not infringe any person's rights, or applicable UK laws. If you discover content in the Research Portal that you believe breaches copyright or violates any law, please contact openaccess@qub.ac.uk.
} 


\title{
A Monte Carlo Simulation Model to Assess Volunteer Response Times in a Public Access Defibrillation Scheme in Northern Ireland
}

\author{
Adele H. Marshall, Karen J. Cairns, Frank Kee \\ Queen's University of Belfast, Northern Ireland, UK. \\ \{a.h.marshall,k.cairns,f.kee\}@qub.ac.uk \\ Michael J. Moore, Andrew J. Hamilton, A.A. Jennifer Adgey \\ Regional Medical Cardiology Centre, Royal Victoria Hospital, Belfast, UK. \\ Andrew.Hamilton@royalhospitals.n-i.nhs.uk \\ moore1@doctors.org.uk \\ Jennifer.Adgey@royalhospitals.n-i.nhs.uk
}

\begin{abstract}
This paper describes the development of a model to assess the distribution of response times for mobile volunteers of a Public Access Defibrillation (PAD) scheme in Northern Ireland. Using parameters based on a trial period, the model predicts that a PAD volunteer would arrive before the Emergency Medical Services (EMS) to $18.8 \%$ of events to which they are paged in a given year period. This is in agreement with what has actually been observed during the trial period (where volunteers have actually reached $15 \%$ of events before the EMS), and thus assisting validation of the model. Results from this model illustrate how ongoing volunteer commitment is key to the success of the scheme.
\end{abstract}

\section{Introduction}

The Northern Ireland Public Access Defibrillation (NI PAD) study is an observational trial that aims to examine the effectiveness and cost-effectiveness of a PAD scheme. PAD schemes aim to reduce the time interval to defibrillation for out-of-hospital Sudden Cardiac Arrest (SCA) victims, through equipping lay volunteers with Automated External Defibrillator (AED) devices. By empowering local communities with PAD, it is hoped more rapid defibrillation can occur for cardiac arrest victims, thus improving patient survival [1] and providing improved patient management. The project is different to any already conducted due to the use of mainly mobile volunteers, in both urban and rural settings.

Data collected from the trial urban/rural districts is being built into a simulation model which is rooted on population statistics, demographic information, and spatial modeling techniques. This model aims to direct investigators towards those variables that are critical to the scheme's success, (which may vary across the geographical regions), and thus ultimately determine how a successful PAD scheme could be implemented across the whole of Northern Ireland in the future.

This paper presents a simulation model for cardiac arrest events within the urban region of North and West Belfast (NWB), in which the response times of the EMS and the PAD volunteers are the key outputs. The Monte Carlo simulation-based model has been developed taking into account both geographic and demographic information for the urban region, together with response-time data collected during the early phase of the trial from within this geographical area. Results from this model are used to assess the impact of volunteer commitment on the scheme's success.

\section{The NI PAD study}

The NI PAD trial is to monitor the effects of PAD in an urban and rural community. The urban region of North and West Belfast spans a geographical area of 60 sq $\mathrm{km}$, with a population of 138591 [2], 47\% of which are male. Based on recent trends, there is expected to be approximately 160 cases of SCA per year [3].

On account of the fact that the majority of cardiac arrest events occur in residential accommodation, the majority of the AEDs deployed in this trial have been to AED-trained volunteers, who carry the packs as they go about their daily business, with only a small portion of AEDs being placed at fixed public locations (such as shopping malls). Training of members of the Police Service of Northern Ireland (PSNI) and members of the public has resulted in around 300 volunteers [4]. 
All mobile volunteers (both members of the public and PSNI) are notified of possible SCA incidents by the EMS through alphanumeric pagers. In the case of the PSNI, AED packs have been placed in patrol cars, and the Police Control Room receives the pages for all possible SCA events in the urban region, thus allowing the controller to alert the nearest available patrol car to attend. Members of the public who volunteer, are expected to carry the AED packs, together with the alphanumeric pagers for a period of one week, at least once during the trial period. A computer based roster scheme has been used to roster the lay volunteers across seven zones [4], (minimizing the mobile volunteers' collective response time to SCA events)

\section{The model}

A model has been produced to assess the impact of the PAD scheme in the urban area of NWB. This model incorporates a number of factors, which will be discussed below.

\subsection{Modeling SCA events}

In the model, SCA events occur at particular Census Output Areas (COAs) (where COAs are the smallest geographical areas for which Census information is available, each comprising around 400 inhabitants). The number of SCA events occurring within any one particular COA is modeled by a Poisson process with the following parameters:

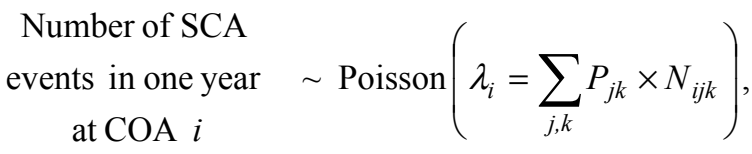

where $P_{j k}$ is the probability of SCA incidents in one year, for a given 5-year age group $j$ and sex $k$, and $N_{i j k}$ is the number of people in COA $i$ from the 5-year age group $j$ and sex $k$. The number of incidents recorded by the World Health Organization's MONICA project in Belfast [5], according to sex and age group over the period 1983-1993, were used to obtain $N_{i j k}$, while population demographic information from the UK Census 2001 [2] for each COA, according to 5-year age groups and sex, was used to obtain $P_{j k}$.

The appropriateness of modeling SCA events as a Poisson process [6] in this urban region was verified through an examination of data collected for SCA events in NWB in 2004. The goodness of fit was assessed by using both the weekly counts of cardiac arrests and the elapsed time between events.
The $\chi^{2}$ goodness of fit statistic for the weekly counts equals 4.18 with 3 degrees of freedom $(p=0.24)$, while the Poisson dispersion test statistic is 64.4 with 51 degrees of freedom $(p=0.10)$. The KolmogorovSmirnov test was used to assess the goodness-of-fit of the continuous exponential distribution to the time interval between SCA events $(p>0.50)$. Figure 1 illustrates the cumulative proportion of observed time intervals against that from the exponential distribution. These results, together with the previous evidence [6] convey high confidence in the null hypotheses that the data is Poisson-distributed.

Note that given the incidence rates in the North and West of the city, the probability that two events will occur within a one-hour period in either area, is much less than $1 \%$.

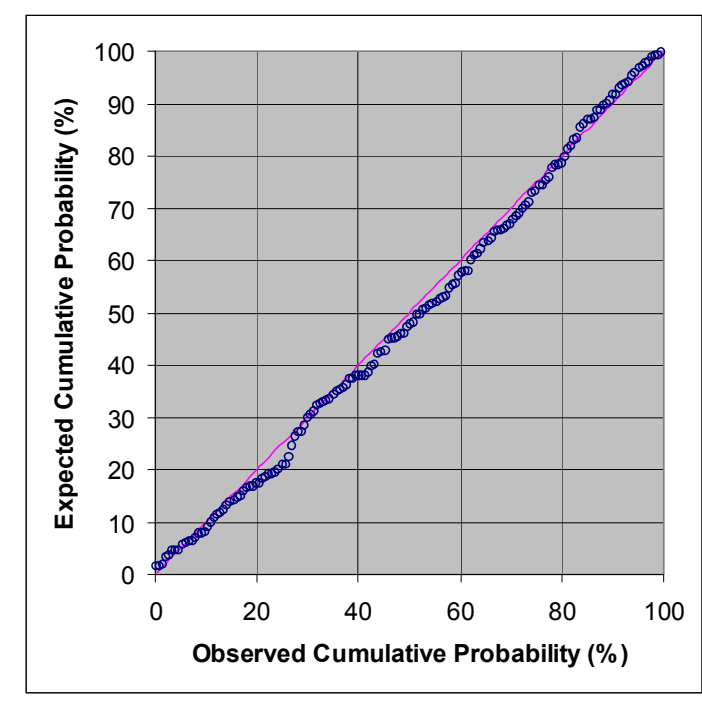

Figure 1. P-P Plot of the cumulative proportion of observed time intervals against that from the fitted exponential distribution.

\subsection{Distribution of EMS response times}

In order to assess the impact of the PAD scheme the response-times of the EMS were obtained for all ambulance-attended events in NWB in 2004. Statistical analyses were performed on this data, and it was found that the electoral ward (an amalgamation of a number of adjacent COAs, usually comprising around 5000 inhabitants) was a significant predictor of the EMS response. The hour of day of an event had only an additional significant effect for particular time-periods (such as around 4am and between 10am and 12 noon), while interaction terms were not significant. 
Due to the significant geographical related variation in the EMS response (see Figure 2), the data was divided according to electoral ward and for each an appropriate distribution was fitted. Such treatment enabled the geographical variation in the EMS response to be incorporated into the model, while, since there was sufficient data available for each electoral ward, it still allowed the suitably of the distribution fits to be assessed.

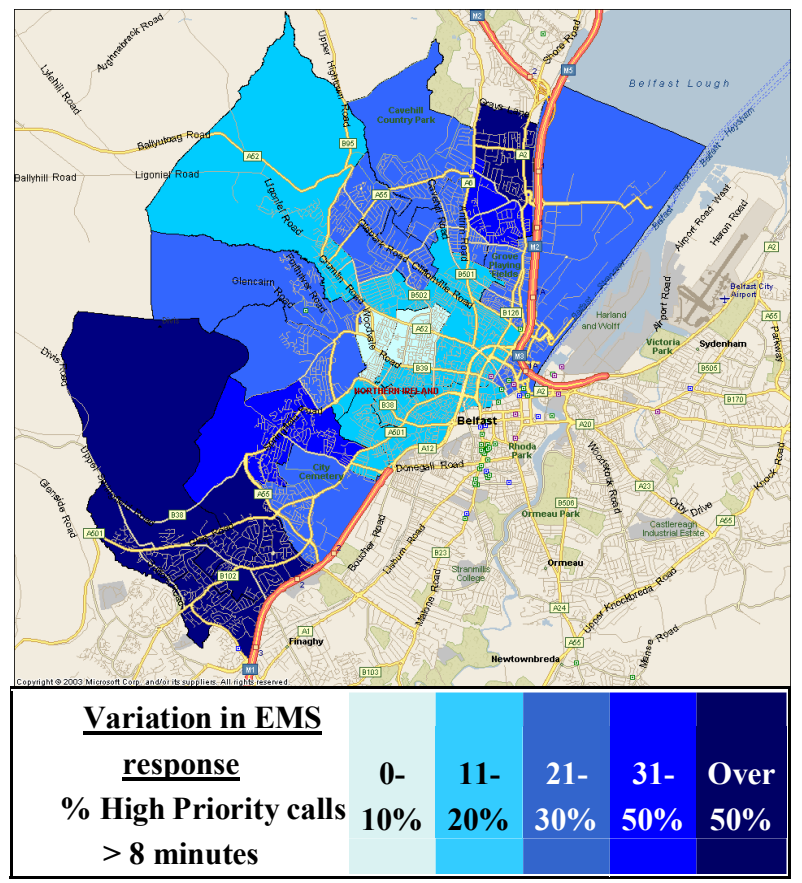

Figure 2. Illustration of variation in EMS response

A number of possible distribution functions were considered including: normal, Erlang, exponential, gamma, inverse Gaussian, lognormal, log-logistic, Pearson Type V, chi-squared, Rayleigh, and Weibull. In each case, the maximum likelihood estimators were obtained for the distribution function and the goodness-of-fit assessed using the KolmogorovSmirnov test (and the Anderson-Darling Test). The $\log$-logistic distribution was found to be an extremely reliable distribution for describing the response times in each of the electoral wards (see Table 1 and Figure $3)$.

The variation in the EMS response times across the city was therefore incorporated through modeling the EMS response-time data from 2004 by electoral ward, via log-logistic distributions, whose parameters were given by the maximum likelihood estimates.
Table 1. Results from testing the hypothesis that the sample data has arisen from the distribution forms indicated

\begin{tabular}{|l|l|}
\hline $\begin{array}{l}\text { Distribution } \\
\text { form }\end{array}$ & $\begin{array}{l}\text { Range of } \boldsymbol{p} \text {-values across the 26 } \\
\text { electoral wards }\end{array}$ \\
\hline Log-logistic & $\begin{array}{l}\text { For 22 of the 26 Wards, } p>0.30, \\
\text { for 4 wards, } 0.10<p<0.30\end{array}$ \\
\hline Erlang & $\begin{array}{l}\text { For 5 of the 26 Wards, } p<0.001, \\
\text { for 6 wards, } p>0.10\end{array}$ \\
\hline $\begin{array}{l}\text { Inverse } \\
\text { Gaussian }\end{array}$ & $\begin{array}{l}\text { For 14 of the 26 Wards, } p<0.001, \\
\text { for 6 wards, } p>0.10\end{array}$ \\
\hline Pearson V & $\begin{array}{l}\text { For 15 of the 26 Wards, } p<0.001, \\
\text { for 5 wards } p>0.10\end{array}$ \\
\hline Gamma & $\begin{array}{l}\text { For 20 of the 26 Wards, } p<0.001, \\
\text { for 5 wards, } 0.001<p<0.01, \text { for } 1 \\
\text { ward, } p>0.50\end{array}$ \\
\hline Lognormal & $\begin{array}{l}\text { For 20 of the } 26 \text { Wards, } p<0.001, \\
\text { for 6 wards, } 0.01<p<0.06\end{array}$ \\
\hline Normal & For all Wards, $p<0.01$ \\
\hline Weibull & For all Wards, $p<0.01$ \\
\hline Rayleigh & For all Wards, $p<0.001$ \\
\hline Chi-Squared & For all Wards, $p<0.001$ \\
\hline Exponential & For all Wards, $p<0.001$ \\
\hline
\end{tabular}

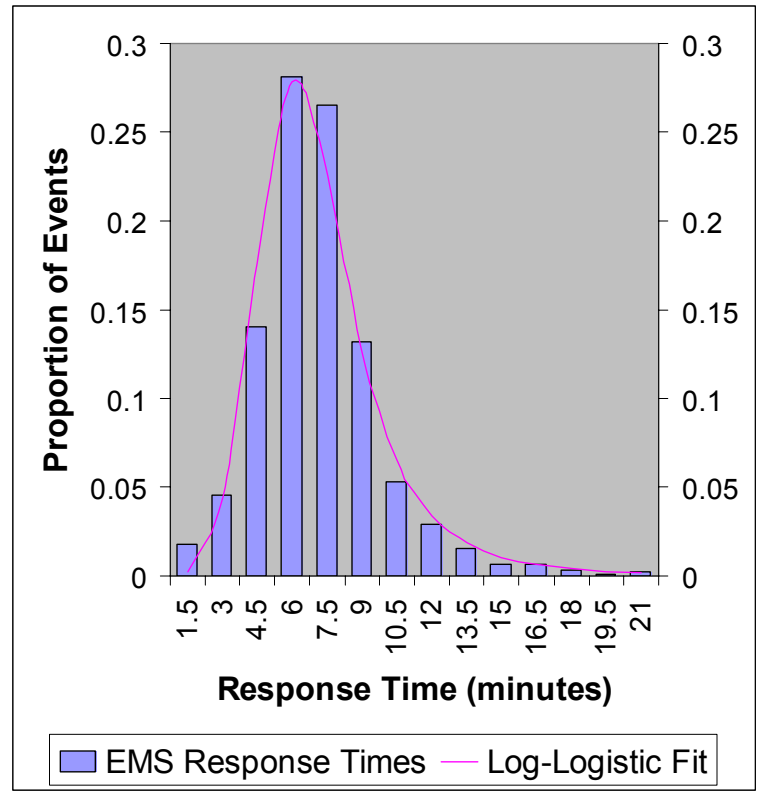

Figure 3. Illustrative log-logistic fit to EMS response time data 


\subsection{Distribution of volunteer response times}

In order to model the response times of the volunteers, road network information was incorporated into the model using Microsoft MapPoint Europe $2004^{\circ}$ together with Visual Basic code, in order to generate a matrix of expected response times for volunteers from the seven zones to each of the 434 COA centroid positions. These expected times were calculated as the shortest travel times between the COA centroids (based on the actual road network), when traveling on the different categorized roads, at the speeds derived from the Northern Ireland Strategic Transport Model (NISTRM) [7] (see Figure 4). The variation in the actual response times (recorded by volunteers during the trial), about these expected response times was modeled using a log-logistic distribution, due to its skewed nature.

Within the simulation model, once a SCA event has been generated at a particular COA, a response is potentially possible from each of the seven lay volunteers on the roster that week, together with the PSNI. However, the model accounts for the fact that not every volunteer will choose to attend a paged event (this could be for several reasons, for example they may not have collected the AED pack to which they were assigned/they may be too far away from the event/ they may be unable to leave the job that they are doing at the time of the page). For example, during the initial period of the trial, it was seen that only $45 \%$ of AEDs packs were collected by volunteers each week, and any individual volunteer was only available to respond to around $30 \%$ of events.

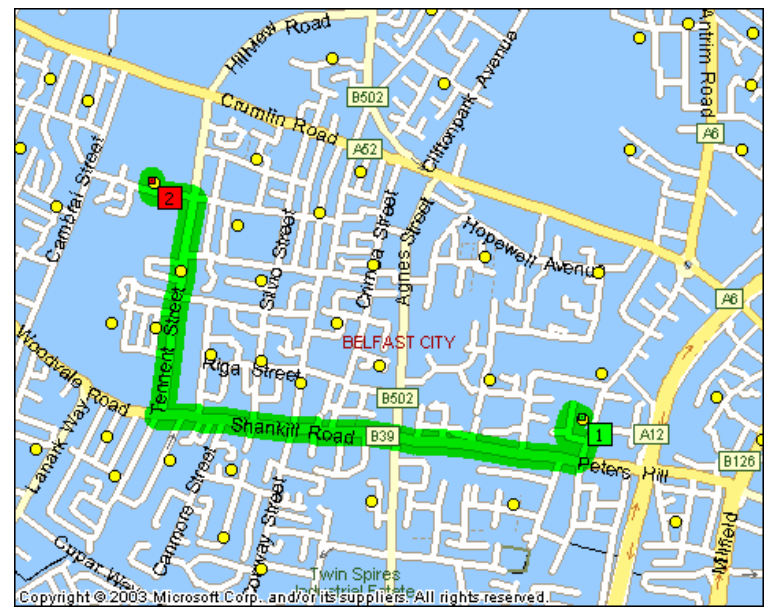

Figure 4. Microsoft MapPoint calculates the shortest route between start/end points, and a time matrix is generated via Visual Basic coding
The response-times of those volunteers that are able to attend a particular event are based on their appropriate expected response times to a particular SCA event's geographical position, together with a random component from the log-logistic distribution.

\subsection{Monte Carlo Simulations}

Modeling the incidence of SCA events as a Poisson process, SCA events were simulated across NWB 360,000 times. For each simulated SCA event, an EMS response time was obtained from the appropriate loglogistic distribution (which depended on the electoral ward of the particular SCA event). The response-times of PAD volunteers to a particular SCA event (including null responses) were calculated based on the geographical position of the simulated SCA event together with the variation seen in the actual responsetime trial data collected.

For each simulated event, the best response from a PAD volunteer was then evaluated, and compared to the EMS response for the same simulated SCA event, in order to generate the best overall response time from either EMS or PAD volunteers to the event. This evaluation thus enables the estimation of the proportion of events where a PAD volunteer arrives before the EMS, as well as the cumulative improvements in the response times due to the combined efforts of the EMS and the PAD scheme, compared to an EMS-only scheme.

\section{Results}

\subsection{Validation of model and assessment of current PAD impact}

Using parameters based on the initial trial period, the model predicts that a PAD volunteer would arrive before the EMS to $18.8 \%(95 \% \mathrm{CI}=13.8 \%$ to $24.7 \%)$ of events to which they are paged in a given year period. This is in close agreement with what has actually been observed during the trial (where volunteers have actually reached $15 \%$ of events before EMS), and so this helps validate the model.

The generated response-times to SCA events can be used to assess the level by which response times will be improved (lowered) if this project was to continue for a longer time period. Based on the initial trial period parameters, the model would suggest that response times would on average be reduced by 56 seconds. These improvements in response times have been quantified in terms of lives saved using Larsen et al. [8]. (Note this is applicable to SCA events where 
the cardiac arrest is witnessed and the initial rhythm of the victim is Ventricular Fibrillation (VF)):

$\mathrm{P}($ survival $)=67 \%-2.3 \% \mathrm{t}_{\mathrm{CPR}}-1.1 \% \mathrm{t}_{\text {defib }}-2.1 \% \mathrm{t}_{\mathrm{ACLS}}$

where:

$-\mathrm{t}_{\mathrm{CPR}}=$ time interval from collapse to Cardiopulmonary Resuscitation,

$-t_{\text {defib }}=$ time interval from collapse to defibrillation,

$-\mathrm{t}_{\mathrm{ACLS}}=$ time interval from collapse to Advanced

Cardiac Life Support.

It was assumed, as in Larsen et al [8], that for EMS personnel, CPR was commenced 1 minute after arrival at the scene followed 1 minute later by defibrillation, while for PAD volunteers, defibrillation occurred 1 minute after arrival at the scene. PAD response times which were less than 30 seconds before the simulated EMS response were not considered to result in any benefit to the victim, since in actual cases where the volunteers could hear an ambulance approaching, the AED was never used by the time the EMS personnel arrived.

Using this formula, together with the cumulative improvements in the responses times to individual events, the model would predict that 1 additional life could be saved per year through PAD (versus the scheme with EMS only attending). However this is based on taking upper estimates for both the proportion of events that are witnessed $(30 \%)$ as well as the proportion of witnessed events where the initial rhythm is VF $(60 \%)$. It has also been assumed that volunteers are dispatched successfully to every SCA event.

\subsection{Impact of volunteer commitment}

Through changing the parameters in the model to account for volunteers' commitment, it has been seen that volunteer availability is a crucial aspect to the success of the scheme. For example, the proportion of events reached before EMS could improve (from 15\% currently) by a further $18 \%$ if their availability to response doubled, and by $32 \%$ if their availability to respond tripled. Furthermore, if every AED was collected each week, this proportion would increase by $23 \%$ (based on volunteer's current levels of availability).

Figure 5 illustrates the improvement in response times for the 7-zone roster, as the proportion of AED packs collected is increased (while keeping the availability of the committed volunteers fixed). Notice that the curve labeled "Best Response" corresponds to the theoretical best response that could result from having this seven zone roster. This graph clearly shows that increasing the commitment levels of the volunteers, even in this 7-zone roster, is crucial in order to dramatically reduce response times.

Based on [8], 3 extra lives could be saved per year, if all 7 AED packs were collected (assuming the availability of the volunteers remains fixed).

The roster model was also regenerated assuming double the levels of volunteers to produce a 14 zone roster (see Figure 6). The proportion of events reached before EMS in this case could improve by a further $19 \%$ (from $15 \%$ ).

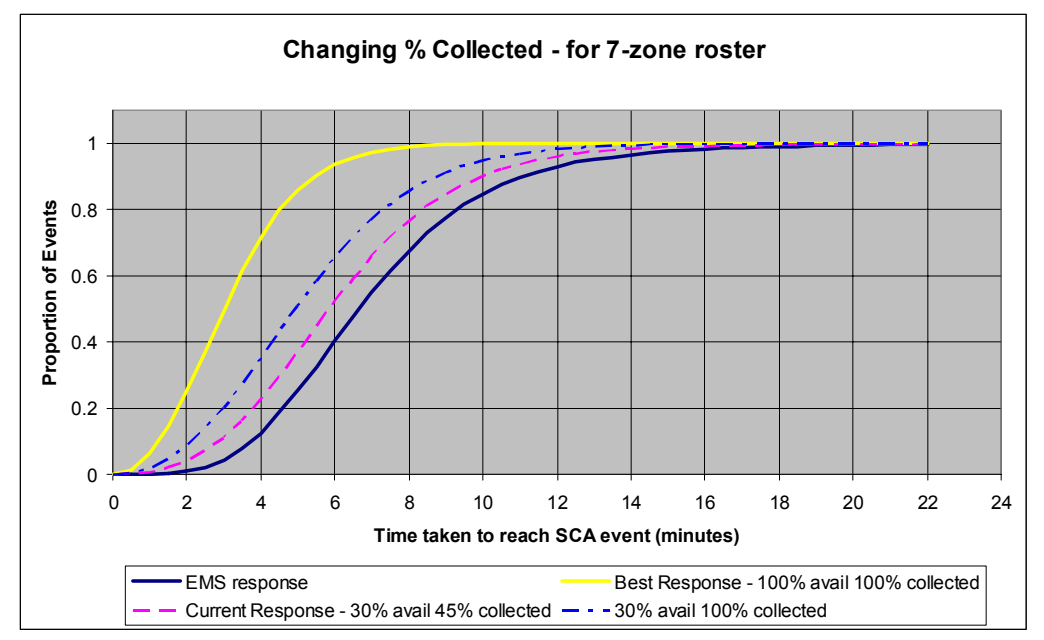

Figure 5. Graph illustrating the improvement in response times through the combined efforts of the EMS and the PAD scheme for a 7-zone roster, and how it varies according to the proportions of AEDs that are collected. 


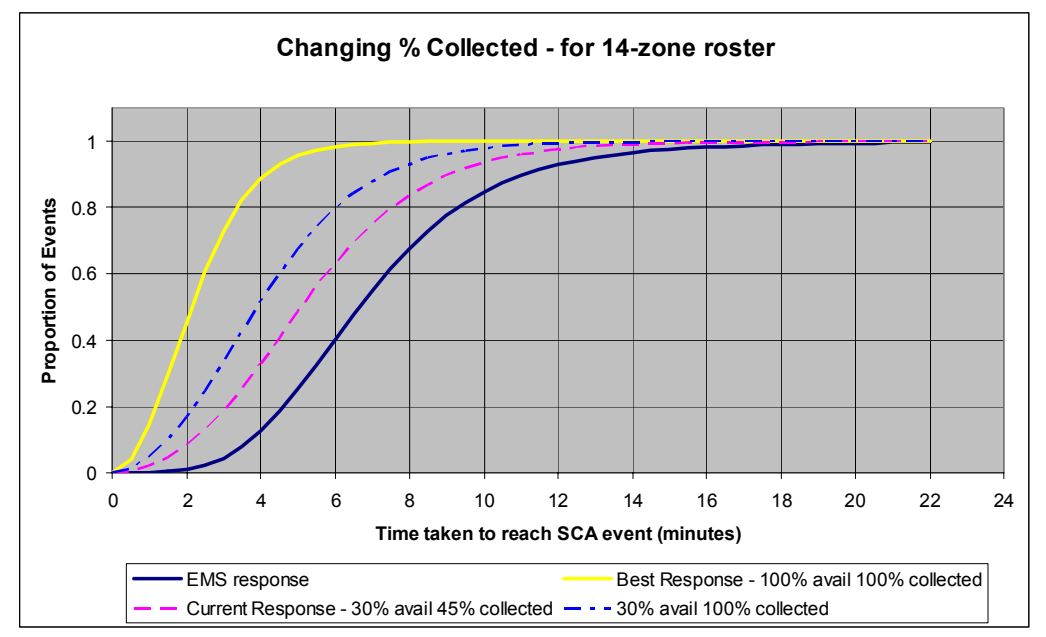

Figure 6. Graph illustrating the improvement in response times through the combined efforts of the EMS and the PAD scheme for a 14-zone roster, and how it varies according to the proportions of AEDs collected.

\section{Conclusion and future work}

A Monte Carlo Simulation model is developed to assess the impact that mobile volunteers make upon the Northern Ireland Public Access Defibrillation Study. The purpose of the study is to reduce the time to defibrillation of a patient who suffers from Sudden Cardiac Arrest and hence increase the survival of patients and their overall management. The simulation model once validated was utilized to assess the impact of volunteer availability and commitment on the scheme.

Once sufficient volunteer data has been collected, the modeling of the volunteer response times will be reexamined. In particular, factors influencing their response times, such as the time of the day, day of week, will be considered, since a volunteer's response in particular, may be more significantly affected by these factors.

A more detailed multiple logistic regression analysis will also be performed for actual SCA event data, in order to incorporate those variables that influence the likelihood a victim will be in a state of witnessed VF (including variables such as age, gender, response time).

\section{Acknowledgements}

This work was supported by a commissioned research grant from the Research and Development Office of the DHSSPSNI (Department of Health, Social Services and Public Safety Northern Ireland). $\mathrm{KJC}$ is also supported through an Engineering \& Physical Sciences Research Council (EPSRC) RCUK
Academic Fellowship. Special thanks go to all the NIPAD team and its collaborators.

\section{References}

[1] H.J.J. Wellens, A.P. Gorgels, and H. de Munter, "Cardiac arrest outside of a hospital: how can we improve results of resuscitation?", Circulation, 2003, 107, pp. 19481950.

[2]http://www.nisra.gov.uk/census/Census2001Output/inde x.html.

[3] M.J. Moore, B.M. Glover, C.J. McCann, et al., "Demographic and temporal trends in out-of-hospital sudden cardiac death in Belfast", Heart, 2006, 92, pp. 311315.

[4] K.J. Cairns, A.H. Marshall, F. Kee, "A Public Access Defibrillation Trial in Urban and Rural Communities in Northern Ireland: Developing the Roster Model", 18th IEEE Symposium On Computer-Based Medical Systems, Proceedings, 2005, pp. 497-502.

[5] H. Tunstall-Pedoe, K. Kuulasmaa, P. Amouyel, et al, "Myocardial infarction and coronary deaths in the World Health Organization MONICA Project. Registration procedures, event rates, and case-fatality rates in 38 populations from 21 countries in four continents", Circulation, 1994; 90, pp. 583-612.

[6] E. Skogvoll, B.H. Lindqvist, "Modeling the Occurrence of Cardiac Arrest as a Poison Process", Ann. Emerg. Med., 1999, 33, pp. 409-417.

[7] Department of Health, Social Services and Public Safety (NI), "Developing better services additional information - average travel speeds in Northern Ireland", 2003.

[8] M.P. Larsen, M.S. Eisenberg, et al., "Predicting survival from out-of-hospital cardiac-arrest - a graphic model", Ann. Emerg. Med., 1993, 22, pp. 1652-1658. 